\title{
Programmer Device
}

National Cancer Institute

\section{Source}

National Cancer Institute. Programmer Device. NCI Thesaurus. Code C50123.

A device designed to write instructions to a memory device. 